\title{
Análisis del impacto de la covid-19 en el turismo y su efecto en la economía del Perú
}

\author{
Melissa Natalia Malpartida Arroyo \\ melissamalpartida2017@gmail.com \\ Universidad Cesar Vallejo \\ Lima - Perú
}

\section{RESUMEN}

OBJETIVO: El objetivo de esta investigación es analizar el impacto de la COVID-19 en el turismo y el efecto que ha generado en la economía del Perú, ya que debido a la pandemia hubo grandes pérdidas de vidas humanas y fuertes pérdidas económicas, lo cual se vio reflejado con el cierre fronteras y la cuarentena obligatoria. METODOLOGÍA: En base a una revisión de información y datos, así como el análisis de una variedad de datos bibliográficos de organismos, tanto de nivel nacional como internacionales, los cuales guardan relación con el tema. RESULTADOS Y CONCLUSIÓN: Los autores consultados indican que se necesita la implementación de estrategias creativas con la finalidad de devolver la confianza a los usuarios y de esta manera promover la llegada de más turistas, ya que la economía se ha visto afectada.

Palabras clave: turismo; economía; inversión; impacto económico; covid-19. 


\title{
Analysis of the impact of covid-19 on tourism and its effect on the Peruvian economy
}

\begin{abstract}
OBJECTIVE: The objective of this research is to analyze the impact of COVID-19 on tourism and the effect it has had on the Peruvian economy, since due to the pandemic there were large losses of human lives and heavy economic losses, which it was reflected with the closure of borders and mandatory quarantine. METHODOLOGY: Based on a review of information and data, as well as the analysis of a variety of bibliographic data from organizations, both national and international, which are related to the subject. RESULTS AND CONCLUSION: The consulted authors indicate that the implementation of creative strategies is needed in order to restore trust to users and in this way promote the arrival of more tourists, since the economy has been affected.
\end{abstract}

Keywords: Tourism, Economy, investment, economic impact, COVID-19.

Artículo recibido: 30 noviembre. 2021 Aceptado para publicación: 29 diciembre 2021 Correspondencia: melissamalpartida2017@gmail.com Conflictos de Interés: Ninguna que declarar 


\section{INTRODUCCIÓN}

Se sabe que el mundo viene siendo golpeado por una crisis sanitaria que ha llevado a muchos países y al nuestro a tomar drásticas medidas debido a la COVID-19, Jacque et al. (2020) precisa que esto se debe a la inamovilidad y el cierre de fronteras, sin embargo, a la fecha el turismo se ha reactivado, pero no genera los mismos ingresos económicos como años atrás.

Ante esta situación la actividad turística se debe de priorizar los protocolos de seguridad para reducir el riesgo de posible contagio entre las personas, esto para salvaguardar la salud y evitar que se sigan propagando los contagios (Tejedor, 2020). El turismo en todos los países es una de las actividades más afectadas económicamente porque implica el desplazamiento de las personas y el ingreso a lugares cerrados para conocer los atractivos turísticos, lo cual estuvo restringido y hasta el día de hoy con un acceso limitado (Ramakumar \& Kanitkar, 2021).

Desde la perspectiva de Marsano (2018) afirma que, en base a las estimaciones del Observatorio Turístico del Perú, se espera que el turismo aumente en un 8,0\%, esto en base a estimaciones esperanzadoras al año 2022, por el contrario, comentarios de otros autores ahora señalan que la contribución del sector turismo al PBI por la pandemia sanitaria se reducirá al 3.66\%. Por tal motivo, Serrano et al. (2020) indica que contrarrestar esta pandemia y el retorno a la normalidad, lógicamente con nuevas normas sanitarias que quedarán como parte de las costumbres de los establecimientos turísticos. La actividad turística se debe de priorizar en la reactivación económica con nuevos protocolos de seguridad que reduzcan al mínimo el riesgo, con el propósito de preservar la salud y también de reestablecer la confianza para que las personas puedan salir de sus viviendas a recorrer lugares turísticos cercanos (Schubert, 2021). Además, se deben incluir medidas que también regulen el tránsito de personas al exterior, será necesario que los países establezcan normas para reabrir sus fronteras (United Nations, 2020). Asimismo, Gutiérrez et al. (2021) indica que el sector turismo en nuestro país ha estado en crecimiento en las últimas décadas para la economía nacional, sin embargo, apreciamos también diversos contextos críticos que presenta el sector y que se viene arrastrando en el Perú, lo cual hasta la fecha no han sido atendidos, o cuando menos, no adecuadamente. 
La ONU (2020) refiere a que existen grandes desafíos que deben resolverse, como la incertidumbre sobre la evolución de la pandemia y la manera en la que se recuperará la confianza en el sector nacional y económico. Se prevé que la economía mundial se contraiga bruscamente, pese a que los gobiernos y entes internacionales han adecuado protocolos para disminuir los efectos socioeconómicos de la COVID-19 (Suárez, 2020). El Perú ha dictado normas para minimizar los contagios dentro del país, siendo algunos: Resolución Ministerial N ${ }^{\circ}$ 112-2020-MINCETUR se establece el Protocolo Sanitario sectorial ante el COVID-19 para agencias de viaje y turismo, Resolución Ministerial $\mathrm{N}^{\circ}$ 115-2020-MINCETUR correspondiente al Protocolo sanitario sectorial ante el COVID19 para albergues, Resolución Ministerial (MINCETUR) N ${ }^{\circ}$ 113-2020-MINCETUR Protocolo Sanitario Sectorial ante el COVID-19 para el guiado turístico, solo así podremos reactivar la economía (Parlamento Andino, 2020).

Así mismo, Ajam (2020) manifiesta que las acciones o medidas que las organizaciones y gobiernos opten deben estar orientadas a recuperar la confianza del consumidor y generar un efecto innovador en la industria, de tal manera que se prime la creatividad y sostenibilidad. Del mismo Min Ho et al. (2021) hacen referencia a que todas las entidades del Estado deben pensar en estrategias que primero se orienten a garantizar el cumplimiento de los protocolos para evitar el contagio, pero a la vez establecer planes de marketing que pueden ser promociones especiales para visitar algunos lugares turísticos, como el proyecto "Vuelve a Perú".

De la Barra et al. (2020) afirma que para la reconstrucción actual del turismo se cuente con el Plan Estratégico Nacional de Turismo, ya que este es un ejemplo para impulsar al sector y posicionar al país como un destino turístico. Cabe señalar que en numerosas ocasiones el país ha sido reconocido mundialmente por su cultura, obteniendo premios por ser un gran referente culinario, maravilla mundial y como destino turístico por excelencia. Por tal motivo PENTUR busca que el país vuelva a ser reconocido, lo cual si bien es favorable para el desarrollo del turismo lo es también para la priorización de la reapertura y el nuevo desarrollo del sector frente a la situación actual que vivimos.

Continuando con Orgaz \& Moral (2017), indican que mayormente los ingresos fuertes de los países en desarrollo son parte de los turistas extranjeros, por tal motivo, resulta una prioridad fortalecer las estrategias para generar ingresos fuertes, sin embargo, las cifras 
que evidenciaba el turismo de antes de la pandemia no serán fáciles de imitar o superar, pero buscar acciones estratégicas contribuirá muchísimo.

Nos indica Gálvez (2021) que para minimizar las afectaciones a raíz de la COVID-19 es indispensable que se empleen estrategias gubernamentales orientadas a proteger la salud de las personas. Cabe señalar que es primordial el cumplimiento de los protocolos, debe hacerse un seguimiento riguroso, ya que siempre existen personas y empresas que buscan "sacarle la vuelta" a la norma, haciendo que la confianza de las personas se vea resquebrajada. Chicaiza et al. (2021) precisa que el Estado tiene la obligación de supervisar las acciones que las empresas turísticas realicen y estas deben seguir los lineamientos del sector, ya que de nada sirve que solo algunas respeten estas normas. La metodología de la actual investigación se con información correspondiente al tema, todo esto mediante una revisión sistemática de buscadores confiables. Asimismo, se utilizaron dos esquemas, el esquema de revisión y el esquema de prisma, los cuales proporcionan un orden y rigurosidad en relación a las fuentes consultadas.

Por otro lado, Rasool et al. (2021) hacen referencia a que la pandemia aún es una incógnita, por ello se debe buscar medidas que puedan ser empleadas en escenarios reales tras el shock sufrido por la actividad turística. Estos escenarios tendrán que redefinirse y evaluarse constantemente en base a la obtención de nuevos datos que aporten a la reducción de perdidas en el sector y contribuya a su desarrollo. Esto significa la participación de grupos de trabajo integrados por especialistas en la elaboración de objetivos estratégicos a corto y mediano plazo frente a la reactivación del turismo. Mendoza et al. (2021) hace referencia a que las medidas a corto plazo serán el impulso para que las empresas turísticas, con ayuda económicas de reactivación, puedan implementar normas de bioseguridad y hacer un seguimiento al cumplimiento de las mismas, sino también analizar el mercado turístico y el movimiento durante la pandemia. Las medidas a mediano plazo se refieren sobre todo a objetivos estratégicos de reactivación y recuperación del sector.

\section{ESTRATEGIAS METODOLÓGICAS O MATERIALES Y MÉTODOS}

\section{Tipo y diseño de investigación}

La actual investigación estudió la variable "Análisis del impacto de la COVID-19 en el Turismo y su efecto en la economía del Perú', la cual tuvo un enfoque cuantitativo 
básico, el cual señala Espinoza (2018), como el que se infiere por el acopio de diferentes datos con el propósito de brindar veracidad para exponer la incógnita expuesta.

Esta investigación se realizó a través de una revisión rigurosa, la cual es un proceso que radica en recolectar artículos para otorgar una vista panorámica de un fenómeno establecido, de forma que con distintas fuentes de datos se pueda desarrollar un resumen cuidadoso y elocuente que aporte a conocer un tema. Este tipo de investigación resulta una manera excelente para recabar y comparar datos (Karunananthan, 2021).

\section{Su esquema se muestra a continuación:}

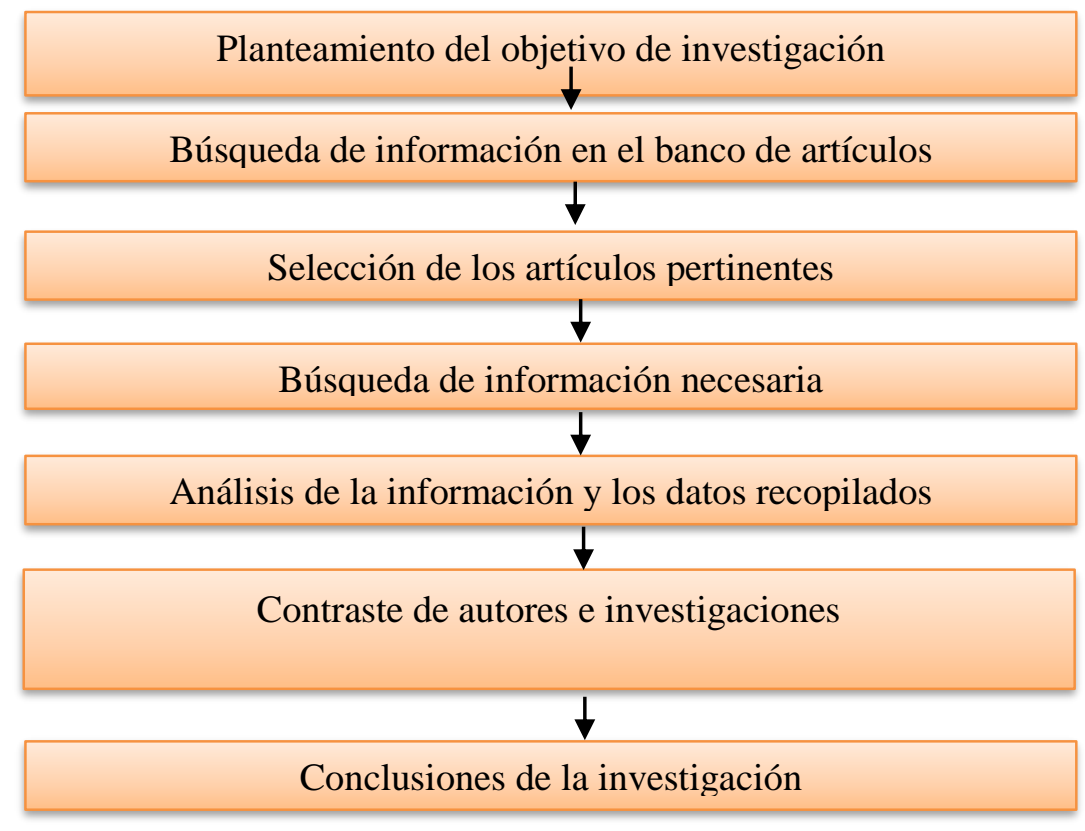

Figura: Esquema de la revisión del artículo

\section{Técnicas e instrumentos de recolección de datos}

Esta investigación se realizó de manera eficaz ya que se recolectó gran cantidad de artículos acerca de análisis del impacto de la COVID-19 en el turismo y su efecto en la economía del Perú; del mismo modo, la recopilación se basó en fuentes de datos confiables como artículos, libros digitales y tesis, a modo que los datos fueron comparados de diferentes autores y países (Palella \& Martins, 2012).

El proceso de la investigación fue mediante una verificación sistemática documental científico, las cuales fueron seleccionadas por tener ciertas características similares, tales como:

- Artículos dentro del rango anual del 2017-2021.

- Artículos escritos en lengua inglés (95\%), español (5\%), portugués (0\%) y otros $(0 \%)$. 
- Selección de artículos en base al título de publicación, presencia de la variable de estudio.

- Clasificación de artículos por resultados y conclusiones similares a los de la investigación.

- Uso de 2 tesis para el contraste de información.

- Selección de 1 libro digitales actuales para el contraste de información.

- Revistas indexadas y confiables.

- Diversidad de autores y países.

\section{Procedimientos}

Los datos alcanzados, forman parte de una revisión sistemática y bibliografía trasparente, las consideraciones para su inclusión fueron el año de publicación, el contenido adecuado en la investigación, y consideraciones como la realidad en las que se desarrollaban y los resultados que los investigadores obtuvieron durante su proceso.

Para la ejecución del estudio se usaron buscadores de internet tales como Scielo, Scopus, Google Académico y repositorios internacionales verídicos como Universidad Nacional Autónoma de México, South Africa journal of Science, para la clasificación de los mismos se usaron las palabras clave como 'COVID-19', 'Tourism', 'Impacts', “'economy', '’Perú impact',

Las investigaciones clasificadas se seleccionaron por ser en su gran mayoría de idioma inglés, portugués y español, además, aquellos que poseían considerable relación con la variable de estudio o que de cierta manera contenían concordancia con los resultados que se querían encontrar. En el buscador Scielo se hallaron artículos que correspondían al idioma extranjero (inglés) 22 y al español 4, los cuales poseían los requisitos requeridos, siendo un total de 26 del mismo modo se recolectaron 8 de Scopus, 3 documentos oficiales de OMS, Naciones Unidas y Unesco, así como de base de datos de Scielo 7 y Google Académico 1.

Los artículos para ser incorporados tuvieron que ser parte de una clasificación severa, artículos pertenecientes a revistas indexadas conocidas y confiables, aquellos que tuvieran objetivos equivalentes a los de esta investigación, resultados que aporten y fueran relevantes. Asimismo, los artículos que tuvieran una diversidad de autores, de modo que otorguen puntos de vista diferentes y contribuyan para dar un panorama claro y real. 
Por otra parte, los artículos que no fueron incluidos para su uso en esta investigación fueron aquellos que no contaban con las palabras clave apropiadas o que no tuvieran conclusiones sin resultados, y menos aquellos que no tuvieran bibliografía veraz, que no correspondieran con el estudio o no aportaran de manera considerable al estudio, del mismo modo, aquellos que no fueran parte del periodo de tiempo determinado o del idioma solicitado.

La selección se realizó en tres filtros: el filtro inicial se clasificó de aquellos artículos que formaran parte de los buscadores requeridos, el segundo filtro fue la lectura rigurosa de las fuentes correspondientes, en el tercer filtro fue la lectura estricta de la parte de metodología, resultados y conclusiones.

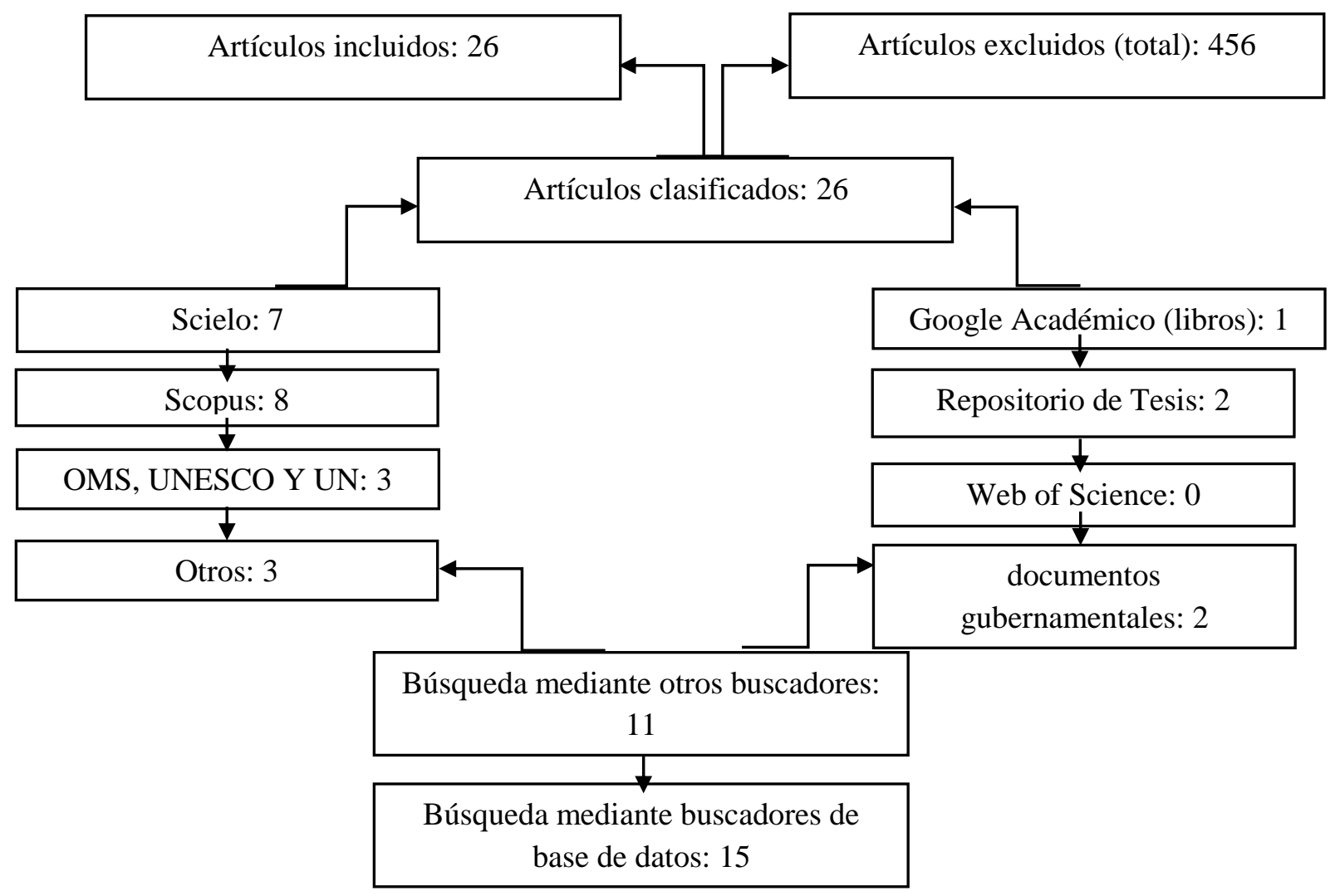

Figura: Esquema PRISMA

\section{RESULTADOS Y DISCUSIÓN}

\section{Resultados}

A raíz de la COVID-19 en todo el mundo se desencadenó un problema que afectó gravemente a la población y a las autoridades, la economía en declive. Como se sabe, muchos países necesitan de las exportaciones y las importaciones para poder subsistir, 
entonces cuando las fronteras se cerraron y la producción bajó, los sectores tuvieron que desarrollar nuevas estrategias para poder mantenerse. Tal es el caso del turismo, este ha sido uno de los sectores más afectados, ya que las personas no podían circular libremente por los distintos lugares turísticos y generar ingresos para el país, simplemente la actividad se detuvo de manera mundial.

La Organización Mundial del Turismo (OMT) evidenció que desde que inició la pandemia más de 100 millones de empleos se perdieron, se debe a que millones de familias dependían de esto para sobrevivir, lo cual posee gran relación con la economía, ya que basta que un sector no genere ingresos para que las ganancias nacionales bajen y consecuentemente afecte a gran parte de la población. Asimismo, es preciso señalar que muchos países en los continentes de Asia y América dependen particularmente del turismo, incluso antes de la pandemia este sector equivalía el 10\% del PBI mundial y el $45 \%$ del empleo en el mundo, cifras que bajaron considerablemente en los últimos meses. En la actualidad muchos gobiernos han adoptado medidas para reactivar el sector, así como implementar estrategias para diversificarlo y darle una mejor sostenibilidad, sin embargo, conocer estos resultados tomará tiempo, ya que los gobiernos deberán resguardar la salud de los ciudadanos, pero también resguardar los empleos y el funcionamiento de las empresas.

Además, las diversas fuentes consultadas indican que los países como Alemania, Reino Unido y Francia se recuperarán más fácilmente porque poseen a gran cantidad de personas vacunadas, sin embargo, no se espera que se llegue a los niveles de afluencia turística ya obtenidos, al menos no hasta el año 2023, ya que las pérdidas que ha tenido el turismo y consecuentemente la economía son mayores de las que se esperaba tener.

En el caso del Perú, es considerado dentro de las naciones con un ingreso medio-alto entre los años 2010-2019, gran parte debido a los ingresos obtenidos del turismo. Las cifras estiman que en el año 2019 se produjeron cerca de 48.9 millones de viajes con fines turísticos y generó 679 mil empleos, es decir, la demanda turística que posee el Perú es alta. Pese a que el Perú y las demás naciones vienen subsanando estos problemas, con las nuevas variantes del virus la situación podría complicarse más debido a la falta de dinamismo económico y la falta de planes "salvavidas" que no se consideraban antes. ¿Como se mencionó, el gobierno dictó distintas medidas para que el sector económico y turístico dejara de tener tantas pérdidas, entre ellas subvenciones a favor de los guías de 
turismo, transferencia de 32 millones de soles al Mincetur para financiar y reactivar el sector, creación de campañas y acciones como “'y tú que planes? ", más de 800 millones en empleos temporales, bonos, apoyo a micro empresas, entre otros.

El turismo nos solo consiste en el descenso en pasajes y visitas a sitios, sino el descenso del ingreso en gastronomía en un 48.2\%, alojamientos cayó en un 43\%, restaurantes en un $47 \%$, quioscos turísticos en un $93.3 \%$, buses de turismo, entre otros sectores. En el año 2019 se recibió 4.4 millones de viajeros en las distintas regiones del país, esto se evidenciaba en las cifras de la Cámara Nacional de Turismo del Perú, asimismo, cerca de 800 mil empleos se perdieron, 200 mil millones de dólares y el PBI que se mantenía en un 3.6\% también bajó con la aparición de la pandemia.

El Perú registró la mayor perdida en ganancias en la historia, pese a que actualmente ya se observa una recuperación en algunas regiones del país, siendo 300 destinos turísticos que ya se encuentran certificados con protocolos de bioseguridad. Es preciso señalar que el turismo es un factor social que afecta la vida de un país entero, la población debe entender que esta pandemia es una oportunidad para desarrollar la visita a espacios turísticos con medidas de cuidado y prevención, como el uso de mascarillas, evitar la aglomeración y espacios cerrados, uso de elementos de limpieza como el alcohol, gel desinfectante, entre otros.

\section{Discusión}

Sánchez et al. (2021) en su artículo " Percepción empresarial de la pandemia por COVID-19 y su impacto en el turismo: un análisis cualitativo del destino Extremadura, España", tuvieron como objetivo principal el análisis de la apreciación que tienen los negociantes del turismo ante el inminente peligro de la covid-19; es por ello que se desarrolló una metodología con enfoque cualitativo, el cual les permitió dar estudio a 122 cuestionarios; consiguientemente brindado como resultado que la emergencia sanitaria global, afecto de gran impacto en el sector turístico y por ende en la economía, más aun si hay lugares de visita clausurados como lo son las reservas nacionales o el decreciente ingreso en los días festivos. Por lo tanto, estos autores tienen semejanzas con la variable de la presente investigación, teniendo como tema primordial el turismo, impacto económico y la covid-19 respectivamente en su país.

Sigala (2020) en su artículo en su artículo 'Tourism and COVID-19: Impacts and implications for advancing and resetting industry and research', el cual presenta como 
objetivo general dar revisión exhaustivamente a la comprensión y gestión de los impactos relacionados al turismo, así como la capacidad de transfigurar con la COVID-19. es por ello que este autor usó como metodología de revisión sistemática, la cual le permitió obtener como efecto, la creatividad de las agencias turísticas o gobiernos, con la intención de hacer más atractivo sus destinos turísticos, en la cual se emplean ofertas, bolsas de viaje, hoteles, sitios recreacionales, etc.; es por ello que se dice que la COVID-19 desde una perspectiva positiva, ha permitido explotar la creatividad de los empresarios y promover el turismo en diversos países logrando recuperarse de la crisis vivida en el 2020. Guliz \& Akbiyik (2020) presentó "Impacts of COVID-19 on global tourism industry: A cross-regional comparison", contiene como objetivo inicial exponer los comentarios de los viajeros en el transcurso de sus viajes en el momento de emergencia sanitaria global; asimismo se desarrolló metodológicamente bajo un enfoque cualitativo permitiendo arrojar como resultado que el área turística se afectó fácilmente por la crisis sanitaria, ya que muchos viajeros han tenido problemas con retraso de vuelos, cancelaciones de los boletos incluso, la cancelación de sus viajes, esto debido a muchas veces en trámites engorrosos para el debido reembolso de su dinero invertido, es por ello que la autora propone contar con un seguro de viaje ante cualquier emergencia ya sea de trámite o de salud, ya que tener un respaldo de viajes permite hacer turismo de un modo más confiable. Abbas et al. (2021) en su investigación en el artículo de revisión sistemática titulado "Exploring the impact of COVID-19 on tourism: transformational potential and implications for a sustainable recovery of the travel and leisure industry", manifiesta que las estrategias planteadas para reestablecer el sector de viajes, en donde la COVID-19 ha implantado desafíos en los empresarios y países, primando la salud de los ciudadanos. Es por ello que la autora estudió las consecuencias y el contexto emergido por la emergencia sanitaria, estableciendo que las empresas turísticas deben de considerar y transformar los sistemas de organización a través de la retroinformación del sector turístico mediante sistemas de entretenimiento, así como medidas de salubridad y distanciamiento para potenciar la calidad de servicio con el valor agregado de salvaguardar la salud del viajero. Lakshmi et al. (2021), en su artículo de investigación "Impact assessment of lockdown amid COVID-19 pandemic on tourism industry of Kashmir Valley, India", manejó como objetivo primordial analizar el impacto del covid-19 en el turismo, teniendo como metodología el enfoque cualitativo, permitiéndole obtener como resultado del estudio 
sobre los responsables de crear medidas provisorias del contagio, en donde al limitar el aforo de los lugares concurridos, limitar el acceso de ciertos países entre otros, generan un gran déficit económico en las empresas turísticas, ya que aun empleando las medidas de salubridad requeridas por cada gobierno, siguen sin sincronizarse para lograr un turismo exitoso; es por ello que los autores proponen emplear una política gubernamental que contenga planificación y determinación para salvaguardar los sitios turísticos como la salud de quienes los visitan.

\section{CONCLUSIÓN O CONSIDERACIONES FINALES}

\section{Conclusiones}

- Como primera conclusión, existe una relación directa entre turismo y la economía, ya que ambos han sido los más afectados por la COVID-19, en especial en zonas que tienen al turismo como fuente principal de ingresos.

- Como segunda conclusión, la reactivación económica del turismo va a depender de los proyectos y programas que apliquen las empresas para brindar al usuario seguridad, de tal manera que puedan acudir a centros turísticos sin temor a contagiarse de la COVID-19.

- Como tercera conclusión, muchos países no estaban preparados para una pandemia de esta magnitud, y por eso se han visto económicamente. Esto ha ocasionado que los países opten por medidas estrictas que favorezcan y contribuyan a reactivar sus sectores que por tanto tiempo se encontraban inactivos, si bien ha habido una gran mejora, no en todos se ha podido restablecer al país.

\section{Recomendaciones}

- Como primera recomendación, el uso de la tecnología puede ayudar a ofrecer experiencias similares a las visitas guiadas presenciales, siendo esta una manera creativa de generar ingresos,

- Como segunda recomendación, se deben implementar sorteos webs que ofrezcan al público descuentos en sus próximas visitas, ya que las personas querrán participar con tal de conocer nuevos lugares, y más aún si obtendrán un descuento o viaje gratis.

- Como tercera recomendación, se aconseja la implementación de programas preventivos ante alguna posible nueva pandemia, en la que el gobierno cuente estrategias para evitar que la economía se vea afectada como ahora. 


\section{REFERENCIAS BIBLIGRAFICAS}

Abbas, J., Terhemba, P., Raza, S., \& Mamirkulova, G. (2021). Exploring the impact of COVID-19 on tourism: transformational potential and implications for a sustainable recovery of the travel and leisure industry. Current Research in Behavioral Sciences, 2, 1-11. doi:https://doi.org/10.1016/j.crbeha.2021.100033

Ajam, T. (2020). More eyes on COVID-19: Perspectives from Economics - The economic costs of the pandemic - and its response. South African Journal of Science, 116(7), 1-2. doi:http://dx.doi.org/10.17159/sajs.2020/8490

Chicaiza, L., García, M., \& Urrea, I. (2021). Economy or health? A Global Analysis of the COVID-19 Pandemic. Journal of Institutional Economics, 23(44), 171-194. doi:https://doi.org/10.18601/01245996.v23n44.08.

De la Barra, A., Bocanegra, S., \& Mayo, J. (2020). Los efectos economicos sobre el turismo receptivo, la exportación de hidrocarburos y de la industria minera a causa del COVID-19 en el Perú. Universidad San Ignacio de Loyola. Obtenido de

http://repositorio.usil.edu.pe/bitstream/USIL/10372/1/2020_De\%20La\%20Barra $\%$ 20Contreras.pdf

Espinoza, E. (2018). Variables and their operationalization in educational research. Part I. Conrado, 14(1), 39-49. Obtenido de http://scielo.sld.cu/scielo.php?script=sci_arttext\&pid=S199086442018000500039

Galvez, A. (2021). Economy, health and COVID-19. (34). Obtenido de http://scielo.sld.cu/scielo.php?pid=S199635212021000100020\&script $=$ sci_abstract\&tlng=en

Guliz, N., \& Akbiyik, A. (2020). Impacts of COVID-19 on global tourism industry: A cross-regional comparison. Tourism Management Perspectives, 36. doi:https://doi.org/10.1016/j.tmp.2020.100744

Gutiérrez, A., Pinares, M., Salazar, W., \& Guerrero, J. (2021). Impacto económico en la calidad de los servicios de salud en el marco de la pandemia COVID-19 en el Perú. Revista de la Facultad de Medicina Humana, 21(1), 248-250. doi:http://dx.doi.org/10.25176/rfmh.v21i1.3244 
Jacque, C., Tarrillo, L., Ramos, J., Pasapera, G., \& Vásquez, L. (2020). Economic and health effects during quarantine by Covid-19 in the peruvian population in 2020 . Revista de la Facultad de Medicina Humana, 20(4), 630-639. doi:http://dx.doi.org/10.25176/rfmh.v20i4.3067

Karunananthan, S. (2021). When is systematic review replication useful, and when is it wasteful? Letter to the editor(45). doi:https://doi.org/10.26633/RPSP.2021.11

Lakshimi, A., Jamal, S., \& Suhail, W. (2021). Impact assessment of lockdown amid COVID-19 pandemic on tourism industry of Kashmir Valley, India. Research in Globalization, 3, 1-13. doi:https://doi.org/10.1016/j.resglo.2021.100053

Marsano, J. (2018). The economic impact of tourism in Peru. Revista UNESCO, 1-11. Obtenido de http://catedraunesco.usmp.edu.pe/wpcontent/uploads/2019/06/impacto-economico-turismo-peru.pdf

Mendoza, R., Almaraz, I., \& Demmier, M. (2021). Impact of COVID-19 on the world economy. XIV Congreso, 14(14), 1-17.

Min Ho, C., Tan, T., Le-Phuc, H., \& Hong, D. (2021). Does the COVID-19 pandemic matter for market risks across sectors in Vietnam? Heliyon, 7(12). doi:https://doi.org/10.1016/j.heliyon.2021.e08453

Orgaz, F., \& Moral, S. (2017). ourism as a potential motor for the economic development of border areas in developing. A case study. . (31). Obtenido de http://www.scielo.org.mx/scielo.php?pid=S1870$90362016000200008 \&$ script $=$ sci_abstract $\&$ tlng $=$ en

Palella, S., \& Martins, F. (2012). Metodología de la Investigación Cuantitativa (Tercera edición). En FEDUPEL (Ed.). Caracas, Venezuela.

Parlamento Andino. (2020). Principales medidas adoptadas por el gobierno preuano frente a la emergencia provocada por la COVID-19. Parlamento andino, 1-67. Obtenido de https://www.parlamentoandino.org/images/actualidad/informescovid/Peru/Principales-medidas-adoptadas-por-el-gobierno-peruano.pdf

Ramakumar, R., \& Kanitkar, T. (2021). Impact of Covid-19 pandemic on the Indian economy: a critical analysis1. Investigación económica, 80(315), 3-32. doi:https://doi.org/10.22201/fe.01851667p.2021.315.76845 
Rasool, H., Maqbool, S., \& Tarique, M. (2021). The relationship between tourism and economic growth among BRICS countries: a panel cointegration analysis. Future Business Journal, 7(1). doi:https://doi.org/10.1186/s43093-020-00048-3

Sánchez, M., Rodríguez, M., \& Ricci, A. (2021). Business perception of the COVID-19 pandemic and its impact on tourism: A qualitative analysis of the Extremadura, Spain, destination. Estudios Gerenciales, 37(159), 265-279. doi:https://doi.org/10.18046/j.estger.2021.159.4427.

Schubert, S. (2021). COVID-19: Economic Consequences for a Small Tourism Dependent Economy. Revista brasilera de Pesquisa en Turismo, 15(1), 1-27. doi:https://doi.org/10.7784/rbtur.v15i1.2297

Serrano, A., Eguía, A., Ruíz, A., Olmo, V., Segura, A., Barquilla, A., \& Morán, A. (2020). COVID-19. History repeats itself and we keep stumbling on the same stone. Medicina de Familia: SEMERGEN, 46(1), 48-54. doi:https://doi.org/10.1016/j.semerg.2020.06.008

Sigala, M. (2020). Tourism and COVID-19: Impacts and implications for advancing and resetting industry and research. Journal of Business Research, 117, 312-321. doi:https://doi.org/10.1016/j.jbusres.2020.06.015

Suárez, M. (2020). Economic and labor impacts generated by covid-19 pandemic in different industries in Uruguay: case studies. Revista Investigación y Negocios, 13(22), 6-22. Obtenido de http://www.scielo.org.bo/scielo.php?pid=S2521$27372020000200002 \&$ script $=$ sci_abstract\&tlng=en

Tejedor, J. (2020). The Social and Economic Impacts of the COVID-19 Pandemic in Latin America. Revista Finanzas y Política Económica, 12(2), 329-334. Obtenido de http://www.scielo.org.co/scielo.php?pid=S2248$60462020000200329 \&$ script $=$ sci_arttext $\&$ tlng=en

United Nations. (2020). The impact of COVID-19 on Latin America and the caribbean. $1-25$. 